\title{
La Violencia y los cambios de la conflictividad en la frontera antioqueña: los casos del Nordeste y el Magdalena Medio, 1948-1958
}

Resumen: Este artículo analiza el fenómeno de La Violencia en Antioquia, Colombia, durante el periodo 1948-1958; en él se argumenta que, si bien tiene un origen multicausal, el proyecto de homogeneización racial y control social que la élite local intentó desplegar en el departamento se convirtió en el factor más relevante del conflicto. Efectivamente, los antecedentes recogidos tanto de fuentes documentales como impresas indican que este proyecto identificó al liberalismo, y en especial al gaitanismo, como las manifestaciones políticas de la barbarie social y racial. En este contexto, la muerte de Jorge Eliécer Gaitán incrementó la violencia bipartidista, adquiriendo un creciente carácter aniquilador.

Palabras clave: violencia, raza, gaitanismo, liberales, conservadores.

\section{La Violencia and changing conflict on Antioquia's borderlands: the Northeast and Magdalena Medio cases, 1948-1958}

Abstract: This paper analyzes the Violencia phenomenon in Antioquia, Colombia during the period 1948-1958. It argues that even though this was a problem of multi-causal origin, the project of racial homogenization and social control that the local elite tried to deploy in the province became the most relevant factor of the conflict. Indeed, the background evidence gathered from both documentary and printed sources indicate that this project identified liberalism, especially Gaitanism, as the political manifestation of social and racial barbarism. In this context, Jorge Eliécer Gaitán's death increased the bipartisan violence, which gained a growing annihilation character.

Keywords: violence, race, gaitanismo, liberals, conservatives.

\section{La Violencia e mudanças do conflito na fronteira de Antioquia: os casos do Nordeste e do Madalena Medio, 1948-1958}

Resumo: Este artigo analisa o fenômeno conhecido como La Violencia na região de Antioquia na Colômbia, no período de 19481958. Argumenta-se que, ainda que fosse um fenômeno de origem multicausal, o projeto de homogeneização racial e controle social que as elites locais tentaram implantar no departamento tornou-se o fator mais relevante do conflito. Os antecedentes coletados de fontes documentais, como impressos, indicam que esse projeto identificou o liberalismo, e especialmente o gaitanismo, como manifestações políticas da barbárie social e racial. Neste contexto, a morte de Jorge Eliécer Gaitán incrementou a violência bipartidária, a qual adquiriu um crescente caráter aniquilador.

Palavras-chave: violência, raça, gaitanismo, liberais, conservadores.

Cómo citar este artículo: Diana Henao Holguín e Igor Goicovic Donoso, "La Violencia y los cambios de la conflictividad en la frontera antioqueña: los casos del Nordeste y el Magdalena Medio, 1948-1958”, Trashumante. Revista Americana de Historia Socia/ 18 [2021]: 212-235.

DOI: 10.17533/udea.trahs.n18a10

Fecha de recepción: 30 de enero de 2020

Fecha de aprobación: 21 de enero de 2021

Diana Henao Holguín: Doctora en Historia por la Universidad de Santiago de Chile [Chile]. Profesora adjunta de la misma universidad. ORCID: 0000-0002-7821-8865.

Correo electrónico: diana.henaoh@usach.cl

Igor Goicovic Donoso: Doctor en Historia de la Universidad de Murcia [España]. Profesor titular de la Universidad de Santiago de Chile [Chile]. ORCID: 0000-0003-2183-7195.

Correo electrónico: igor.goicovic@usach.cl 


\title{
La Violencia y los cambios de la conflictividad en la frontera antioqueña: los casos del Nordeste y el Magdalena Medio, 1948-1958*
}

\author{
Diana Henao Holguín e Igor Goicovic Donoso
}

\section{Introducción}

Este artículo pretende ahondar en un tema ampliamente estudiado por la Chistoriografía colombiana: La Violencia. Podría considerarse que la historia de la violencia política en Colombia inicia con este fenómeno, que se dio entre 1946 y 1965, cuando el país entró en un momento de crisis permanente. Como se verá, el análisis de este conflicto tuvo importantes avances en la década de los ochenta, cuando empezaron a plantearse estudios a nivel regional, tendencia que fue desarrollada gracias al reconocimiento de que La Violencia tuvo escenarios y periodizaciones particulares de acuerdo con el contexto en el que se dio. Siguiendo esta línea, el artículo plantea un análisis regional, particularmente en las zonas fronterizas ubicadas al oriente del departamento de Antioquia. Se busca ahondar en dos preguntas relacionadas con la configuración estatal: ¿cómo se dio la intervención del Estado a nivel departamental en las zonas de frontera del Magdalena Medio y el Nordeste antioqueños durante el periodo previo a 1948? y ¿cuáles fueron los cambios a partir de ese año? Para ello, se establecerán tres momentos distintos a lo largo de esa década. En primer lugar, el estallido que se da en la región tras la muerte de Jorge Eliécer Gaitán y las expresiones que tuvo el Bogotazo a nivel local. ${ }^{1}$ En segundo lugar, la proliferación de bandas de guerrilleros liberales en la región y los enfrentamientos con ejércitos privados de conservadores, así como la persecución de campesinos liberales y conservadores. Por último, a partir de 1953, la presencia militar en la frontera, que se hace mayor bajo la dictadura de Gustavo Rojas Pinilla.

* Este artículo forma parte del proyecto de investigación: USA1799,VRIDEI 031952IG_GO. Universidad de Santiago de Chile.

1. El Bogotazo fue una protesta social ocurrida a causa de la muerte de Jorge Eliécer Gaitán el 9 de abril de 1948. Los disturbios que se dieron en un primer momento en Bogotá se extendieron a otras ciudades y pueblos del país y fue uno de los detonantes de La Violencia. 
La hipótesis que guía este trabajo señala que la conflictividad durante el periodo de estudio no fue solo el resultado de la lucha por los recursos naturales en un contexto de configuración territorial, sino también de la conformación de las redes de poder local en zonas predominantemente liberales. Por tanto, hay una multicausalidad en la aparición de La Violencia, momento en el cual la intervención desde el centro del departamento cambia radicalmente. Asimismo, se plantea, en seguimiento de la línea propuesta por Mary Roldán y retomada por Daniel Tubb, ${ }^{2}$ que la violencia puede observarse desde una perspectiva racial que enfatiza en la mirada que se tiene desde las zonas céntricas del departamento hacia los espacios fronterizos y sus dinámicas políticas y culturales.

Según Ingrid Bolívar, el Estado en Colombia no es una entidad monolítica ni homogénea y su relación con la sociedad depende de cómo se resuelven las relaciones conflictivas con las redes de poder regionales y locales que median entre ambos. ${ }^{3}$ De esta forma, se puede comprender mejor la manera en que las zonas céntricas de Antioquia intervinieron durante la primera mitad del siglo XX en las zonas de frontera ubicadas al oriente del departamento y cuáles fueron los mecanismos que utilizaron las élites políticas y comerciales para tratar de integrar estos territorios al centro.

\section{Tendencias de la historiografía de La Violencia en Colombia}

La violencia ha sido un elemento constitutivo de las sociedades latinoamericanas. Durante la segunda mitad del siglo XX, la violencia política fue un recurso desplegado en situaciones de dictadura, pero también en los momentos en que los regímenes democráticos se mantuvieron estables. A pesar del papel decisivo de la violencia en la conservación o modificación del orden social, la investigación histórica en torno a este tema en América Latina sigue siendo deficitaria. Si bien ha habido avances importantes desde la sociología y la ciencia política, especialmente en torno a las organizaciones y movimientos revolucionarios de la década de los cincuenta y hasta los años setenta, es necesario ampliar y profundizar el análisis acerca del uso y el papel de la violencia en los procesos históricos. Asimismo, resulta relevante analizar la forma en que las clases dominantes y los sectores populares han apelado a ella. ${ }^{4}$

Efectivamente, las miradas de conjunto son escasas, y esto constituye una paradoja, ya que las condiciones estructurales en las cuales se despliega la violencia

2. Mary Roldán, A sangre y fuego. La Violencia en Antioquia, Colombia, 1946-1953 (Bogotá: Instituto Colombiano de Antropología e Historia / Fundación para la Promoción de la Ciencia y la Tecnología, 2003); Daniel Tubb, "Narratives of citizenship in Medellín, Colombia", Citizenship Studies 17.5 (2013): 627-640, doi: 10.1080/13621025.2013.818380.

3. Ingrid Bolívar, Violencia política y formación del Estado. Ensayo historiográfico sobre la dinámica regional de la violencia de los Cincuenta en Colombia (Bogotá: Universidad de los Andes / CINEP, 2003) 15.

4. Waldo Ansaldi y Verónica Giordano, coords., América Latina. Tiempos de violencias (Buenos Aires: Ariel, 2014). 
son compartidas: explotación laboral, pobreza, exclusión, discriminación y represión, pero las especificidades nacionales y regionales, los procesos históricos diferenciados, así como la heterogeneidad en la composición étnica y cultural de la población, establecen puntos de distinción que resisten las generalizaciones. No obstante, el trabajo de Peter Imbusch y Michel Misse permitió establecer una perspectiva longitudinal respecto del fenómeno, al enfatizar las particularidades regionales del mismo y señalar la centralidad de la violencia en la configuración de las relaciones de poder. ${ }^{5}$ Asimismo, algunas compilaciones recientes han colocado el acento en la comparación de procesos de largo aliento, con el objetivo de establecer los puntos de conexión entre las viejas problemáticas que dieron origen a la violencia (procesos de conquista, colonización, despojo y marginalidad) y la emergencia de nuevas conflictividades asociadas a violencias emergentes. ${ }^{6}$

Corresponde indicar que, en el mismo periodo en que se desarrolló en Colombia el proceso conocido como La Violencia, en otras regiones de América Latina también se desencadenaron acontecimientos violentos. En Chile, la movilización obrera inaugurada en 1946 fue enfrentada por el gobierno con la proscripción del Partido Comunista y el confinamiento de cientos de sus militantes en el campo de prisioneros políticos de Pisagua (1948), situación que se extendería hasta la derogación de la Ley de Defensa Permanente de la Democracia en 1958. En Bolivia, una insurrección popular, liderada por los obreros de las minas de estaño afiliados a la Central Obrera Boliviana (COB) y al Movimiento Nacionalista Revolucionario (MNR), inició en abril de 1952 un proceso de transformaciones económicas (como la nacionalización del estaño y la reforma agraria) y políticas (instauración del sufragio universal) que pusieron fin al régimen oligárquico. En Guatemala, por su parte, el gobierno reformista de Jacobo Arbenz fue derrocado en 1954 por una intervención armada propiciada por el gobierno norteamericano, lo que dio inicio a una sistemática represión contra los simpatizantes de la administración caída. Un año después, en Argentina, el gobierno populista de Juan Domingo Perón era derribado por una intervención militar que, al igual que en Guatemala, persiguió a los partidarios de Perón, pero, a su vez, sentó las bases para una situación de inestabilidad y enfrentamientos que se extendieron hasta la restauración democrática de 1983. Por último, en 1953 un grupo de revolucionarios cubanos intentaron asaltar, infructuosamente, un cuartel militar en Santiago de Cuba. Pese a su fracaso inicial, tres años después, los insurgentes se instalaron en la Sierra Maestra, en la región suroriental de Cuba y desde ahí desplegaron una exitosa lucha guerrillera que

5. Peter Imbusch y otros, "Violence Research in Latin America and the Caribbean: A Literature Review", International Journal of Conflict and Violence 5.1 (2011): 87-154.

6. Carlos Alba Vega y Dirk Kruijt, "Viejos y nuevos actores violentos en América Latina: temas y problemas", Foro Internacional 47.3 (2007): 485-516; Pablo Pozzi y Claudio Pérez, eds., Historia oral e historia política. Izquierda y lucha armada en América Latina, 1960-1990 (Santiago de Chile: LOM Ediciones, 2012) 7-17; Igor Goicovic y JaquelineVassallo, comps., América Latina: violencias en la historia (Valparaíso: América en Movimiento, 2018) 9-17; Dirk Kruijt y otros, eds., Latin American Guerrilla Movements. Origins, Evolution, Outcomes (Nueva York: Routledge, 2020) 1-17. 
puso fin a la dictadura de Fulgencio Batista (1959). El triunfo de las guerrillas del Movimiento 26 de Julio, liderado por Fidel Castro, dio inicio a un nuevo ciclo de violencia política en la región. No obstante, estos temas han tenido un desarrollo historiográfico desigual, escaso en Chile, relativo en Bolivia y Guatemala, y muy amplio y consistente en Argentina y Cuba. ${ }^{7}$

A pesar de estos déficits en la investigación en América Latina, el caso de Colombia resulta paradigmático no solo porque la violencia ha sido desplegada en un contexto democrático, sino que también, y justamente debido a la duración del conflicto armado, existe una importante producción científica sobre el tema. Uno de los fenómenos más estudiados por la historiografía colombiana es La Violencia, que se dio entre 1946 y 1965, y que estuvo caracterizado por la confrontación bipartidista, expresada por medio de hechos violentos, lo que le imprimió rasgos distintivos a este periodo, el cual no se dio de manera homogénea en todo el país, y cuyo desarrollo encubre diversos procesos a nivel regional y local.

Así, el estudio de este fenómeno ha permitido identificar múltiples aristas de entrada al análisis de la violencia desplegada por distintos actores en la segunda mitad del siglo XX, y uno de los trabajos que mejor reúne las perspectivas en la materia fue el que resultó del Simposio Internacional sobre la temática en 1984. En el libro Pasado y presente de la violencia en Colombia se presenta la relación de la violencia con la estructura económica y social del país y los conflictos agrarios. ${ }^{8}$ Asimismo, se hace un análisis de las violencias en plural, en el que se destaca la importancia de la regionalización, se presenta una mirada de larga duración, al vincularla con las guerras civiles del siglo XIX, y, finalmente, se analiza la importancia del gaitanismo, pues las zonas más conflictivas durante La Violencia coincidieron con regiones y localidades donde había un importante grado de adhesión a Jorge Eliécer Gaitán, como se observará más adelante.

Ahora bien, dentro de la amplia historiografia sobre La Violencia interesa destacar dos trabajos. ${ }^{9}$ En primer lugar, el de Paul Oquist, Violencia, conflicto $y$

7. Para el caso chileno véase Viviana Bravo Vargas, "La sangre, la furia y la memoria: Ramona Parra en el martirologio comunista de la postguerra (Chile 1946-1947)", Páginas 9.20 (2017): 32-52. Para el caso boliviano, James Dunkerley, Rebelión en las venas. La lucha política en Bolivia, 19521982 (La Paz: Plural Editores, 2003) 67-114. Sobre la caída del gobierno de Arbenz, Roberto García Ferreira, "La revolución guatemalteca y el legado del presidente Arbenz", Anuario de Estudios Centroamericanos 38 (2012): 41-78. Entre los múltiples trabajos disponibles para el caso argentino destacamos el de Alejandro Cattaruzza, Historia de la Argentina, 1916-1955 (Buenos Aires: Siglo XXI Editores, 2016) 181-250. En relación con la Revolución Cubana véase Rafael Rojas, Historia mínima de la Revolución Cubana (México: El Colegio de México, 2015).

8. Gonzalo Sánchez, "Los estudios sobre la violencia: balance y perspectivas", Pasado y presente de la violencia en Colombia, comps. Gonzalo Sánchez y Ricardo Peñaranda (Bogotá: Fondo Editorial Cerec, 1991) 19-44.

9. Otros trabajos importantes sobre La Violencia: Darío Betancourt y Martha García, Matones y cuadrilleros. Origen y evolución de la violencia en el occidente colombiano, 1946-1965 (Bogotá: Tercer Mundo Editores, 1990); Germán Guzmán Campos, Orlando Fals Borda y Eduardo Umaña Luna, La violencia en Colombia: estudio de un proceso social (Bogotá: Ediciones Tercer Mundo, 1962); 
política en Colombia, en donde se plantea la pregunta por La Violencia a partir de la caracterización del Estado. ${ }^{10} \mathrm{El}$ autor sostiene que esta se dio por el "derrumbe parcial del Estado"; según Oquist, el funcionamiento del Estado colombiano, que se fortaleció en las décadas de los treinta y los cuarenta, se vio entorpecido por el profundo conflicto entre las élites de los dos partidos en que estaban divididas las clases dominantes. Este "derrumbe" se reflejó en el quiebre de las instituciones políticas, la pérdida de legitimidad del aparato estatal, las contradicciones dentro de las fuerzas armadas y la ausencia física del Estado en algunas zonas del país. Esta tesis influyó en trabajos posteriores sobre el tema y abrió nuevas perspectivas explicativas. A su vez, Oquist abonó el terreno para nuevos estudios centrados en el análisis del fenómeno a nivel regional, con el fin de ver las modalidades de la violencia y los tipos de conflictos.

En segundo lugar, está el trabajo de Daniel Pécaut, Orden y violencia: Colombia 1930-1953, que intenta hacer un balance de conjunto para trazar un mapa general de La Violencia. ${ }^{11}$ Pécaut plantea que este fenómeno es una yuxtaposición de violencias irreductibles. Esto significa que bajo el nombre de Violencia "se dan cita un conjunto de fenómenos diferenciables en tiempo y lugar, que a partir de la referencia a una división partidista se extienden desde la resistencia campesina hasta la extorsión económica, sin que por ello puedan derivarse de un único conflicto". ${ }^{12}$ En oposición a la tesis de Oquist, para Pécaut, lo que ocurrió durante La Violencia fue la disolución del Estado, pues, a pesar de que desde la República Liberal este intentó mediar para garantizar el interés general, a principios de la década de los cuarenta el proceso se disolvió y empezaron a primar los intereses de las élites liberales y conservadoras, las cuales no habían permitido delegar el poder real al Estado. En consecuencia, los canales de mediación se disolvieron.

Estos dos trabajos dieron luces para marcar la más reciente tendencia de los estudios sobre LaViolencia, donde se propone analizarla a nivel regional y desplazar los enfoques globalizantes. Ahora bien, el trabajo de Ingrid Bolívar profundiza mucho más la pregunta por la configuración estatal, al sostener que La Violencia sería una expresión de los esfuerzos de integración territorial y social, más que una muestra de la debilidad del Estado. ${ }^{13}$ En consonancia con este planteamiento, Fernán González sostiene que los procesos de violencia pasados y presentes, y la manera en la que se ha construido el Estado colombiano, tienen que ver, en primer lugar, con el poblamiento de las regiones y cómo estas se han configurado e integrado a la

Carlos Miguel Ortiz Sarmiento, Estado y subversión en Colombia. La Violencia en el Quindío años 50 (Bogotá: Cerec, 1985); Gonzalo Sánchez y Donny Meertens, Bandoleros, gamonales y campesinos. El caso de la Violencia en Colombia (Bogotá: Punto de Lectura, 2006).

10. Paul Oquist, Violencia, conflicto y política en Colombia (Bogotá: Biblioteca Banco Popular, 1978).

11. Daniel Pécaut, Orden y violencia: Colombia 1930-1953 (Medellín: Fondo Editorial Universidad Eafit, 2012)

12. Bolívar 43.

13. Bolívar 7. 
nación, ${ }^{14}$ lo que apunta a los procesos de colonización en las fronteras internas, caracterizadas por su alta conflictividad y violencia. Asimismo, la violencia tiene que ver con la forma en que los pobladores se han organizado a nivel local y cómo estos espacios se han ido articulando al Estado. Aquí, los partidos políticos, las redes de poder local y los mecanismos clientelistas han tenido un papel importante. Así, el análisis regional ha marcado los trabajos más recientes sobre el tema en las últimas décadas.

Los estudios que abordan LaViolencia en Antioquia buscan explicaciones a través de la forma en que distintos territorios del departamento buscaron ser anexados a los proyectos regionales, auspiciados por las élites comerciales y políticas de las zonas centrales. En este sentido, el trabajo de Mary Roldán es un referente obligado. Roldán sostiene que el surgimiento de La Violencia no fue el resultado de odios partidistas heredados, sino que más bien fue un catalizador que hizo aflorar otros conflictos locales. Además, fue una lucha fundamental por imponer un proyecto departamental hegemónico; de ahí que en el desarrollo de su explicación sean fundamentales las diferencias religiosas, étnicas y raciales que existen en el departamento, puesto que estas definen la manera en que se desencadenó la violencia. ${ }^{15}$ Este correspondería, según Roldán, a un "proyecto hegemónico regional", el cual se basa en un discurso de la diferencia cultural que jerarquiza los espacios geográficos del departamento y crea visiones del "otro". ${ }^{16}$ De esta manera, se distinguían unas áreas centrales, que corresponden a Medellín y sus zonas aledañas, el Oriente cercano y el Suroeste, y unas zonas periféricas correspondientes a las subregiones del Nordeste, el Bajo Cauca, Urabá y el Magdalena Medio. Las primeras se consideraban las portadoras de los valores morales antioqueños asociados al comercio, la industria, la familia patriarcal, la religión católica, el orden social, el conservadurismo y la pujanza, y las segundas representaban la antítesis de estos valores, por lo que eran consideradas violentas y bárbaras. ${ }^{17}$ Cabe anotar aquí que la construcción de la nación colombiana ha estado marcada por un proceso de jerarquización y racialización de las regiones, donde las zonas centrales, que desde la época colonial estuvieron articuladas a la metrópoli, son asociadas al progreso, y las zonas periféricas, que corresponderían a las zonas de frontera interna, han estado asociadas al atraso y a la violencia. ${ }^{18}$

14. Fernán González, Poder y violencia en Colombia (Bogotá: Odecofi-CINEP, 2014).

15. Roldán, $A$ sangre y fuego 30.

16. Mary Roldán, "Violencia, colonización y la geografia de la diferencia cultural en Colombia", Análisis Político 35 (1998): 5.

17. Estas ideas también fueron desarrolladas por Nancy P. Appelbaum, Muddied Waters: Race, Region, and Local History in Colombia, 1846-1948 (Durham / Londres: Duke University Press, 2003); Claudia Steiner, Imaginación y poder: el encuentro del interior con la costa en Urabá, 1900-1960 (Medellín: Editorial Universidad de Antioquia / Ediciones Uniandes, 2019).

18. Para un análisis sobre la frontera en Colombia véase Margarita Serje, El revés de la nación: territorios salvajes, fronteras y tierras de nadie (Bogotá: Universidad de los Andes, 2011). 
En un trabajo más reciente, Daniel Tubb analiza la contradicción entre ciudadanía y violencia en Antioquia, y afirma que la intervención del gobierno regional buscó mejorar la moral de quienes habitaban las zonas de frontera del departamento. Las narrativas sobre lo moral fueron integradas desde la "antioqueñidad", una identidad regional racializada y espacializada. Es decir, los residentes de las zonas centrales del departamento se veían a sí mismos como más blancos, trabajadores y civilizados, mientras que quienes habitaron las zonas de frontera eran vistos como menos civilizados, perezosos y de moral relajada. El resultado de estas visiones del otro fue el estallido de la violencia. ${ }^{19}$ En este sentido, el estudio de la violencia en Antioquia permite reconocer otras categorías de análisis fundamentales, de manera que ya no solo la confrontación clasista explicaría el conflicto, sino que también la raza permitiría una entrada importante al análisis. Cabe anotar que raza, género y clase se dan de manera interrelacionada en la realidad social. ${ }^{20}$

\section{Breve mirada a las formas de intervención estatal antes de 1948}

Hasta 1948 se desplegaron distintos mecanismos que buscaban la intervención e integración de las zonas marginales al centro del departamento. En primer lugar, hay que advertir que el Magdalena Medio y el Nordeste antioqueños empezaron a ser anexados al centro de manera más sistemática desde la década de los veinte, cuando los avances en las vías de comunicación permitieron que varios empresarios agrícolas de Medellín establecieran haciendas para la cría y ceba de ganado, especialmente en Puerto Berrío. ${ }^{21}$ La consolidación de esas haciendas se hizo por medio de vías legales como el denuncio de tierras baldías y la compra de mejoras; también se usaron vías de hecho para ocupar las tierras, lo que trajo consigo la expulsión de colonos y, consecuentemente, el conflicto. La dinámica de adjudicación de tierras públicas también se dio en Segovia y sobre todo en Remedios cuando en la década de los cuarenta se establecieron algunas haciendas ganaderas. No obstante, desde tiempos coloniales, la principal actividad económica fue la minería; por ello, históricamente, estos pueblos habían sido ocupados por colonos mazamorreros que transitaban entre la agricultura y la minería. Paralelamente, en Segovia se instaló desde finales del siglo XIX la compañía extranjera Frontino Gold Mines, alrededor de la cual se empezó a configurar un movimiento obrero importante.

A la par de la consolidación de la gran propiedad, los gobiernos de turno promovieron la ocupación campesina de tierras públicas. No obstante, en el gobierno

19. Tubb.

20. Para una conceptualización de la categoría de raza y cómo opera en el caso colombiano véase Peter Wade, Gente negra, nación mestiza: dinámicas de las identidades raciales en Colombia (Bogotá: Siglo del Hombre Editores / Ediciones Uniandes, 1997).

21. José Roberto Álvarez Múnera, Mercado, ganado y territorio: haciendas y hacendados en el Oriente y el Magdalena Medio antioqueños (1920-1960) (Medellín: Universidad de Antioquia / Editorial Universidad Pontificia Bolivariana, 2016). 
central había visiones racializadas acerca de quiénes debían ser los campesinos ideales para ocupar las zonas de frontera:

Se [debía] mejorar el elemento humano [...] por medio de la llegada de "agricultores verdaderos" para insertarlos en esa "sociedad viciada" para que en el transcurso del tiempo aparezca una generación mestiza formada dentro de un ambiente propicio y con una cultura acorde con las aspiraciones de transformación que hoy se tienen.22

Otro mecanismo importante de intervención en el ámbito local fue la asignación de alcaldes por parte del gobernador. Sin embargo, el nombramiento de cargos públicos siempre se enfrentó a una serie de dificultades en estos municipios. No hay que olvidar que la remoción de alcaldes fue una constante. En la correspondencia dirigida a la gobernación de Antioquia desde los municipios hay numerosas peticiones de vecinos para cambiar a los alcaldes de turno, quienes no permanecían en el cargo por mucho tiempo. Los alcaldes eran afuerinos y debían negociar y ganar la simpatía de los miembros de los concejos municipales para que sus proyectos fueran aprobados; en cambio, los concejales eran vecinos notables de los pueblos y movían las redes del poder local.

Asimismo, una de las figuras importantes para la fiscalización de los municipios fue la del visitador administrativo. Estos agentes viajaban a los pueblos con el fin de vigilar distintos asuntos a nivel local y no solo observaban, sino que ayudaban a acelerar procesos civiles y penales. En muchas ocasiones, su presencia sirvió para dirimir conflictos entre vecinos. Su labor se hizo más visible a partir de 1938, cuando por decreto se establecieron sus funciones, por lo que debían encargarse de "toda sección departamental o municipal en la cual deban tratarse o solventarse cuestiones de las cuales depende [...] la buena marcha de la Rama Ejecutiva". ${ }^{23}$ Estos visitadores también ayudaron a construir discursos y visiones racializadas de los territorios a los que viajaban. Ejemplo de ello fue la visión que el visitador Guillermo White Uribe refirió en un informe acerca de los habitantes de Segovia en 1945: "no viven santamante y sus costumbres son idénticas a las de las ciudades y pueblos de la costa, donde el amor libre es practicado y tolerado hasta por las autoridades". ${ }^{24}$ Este tipo de discursos no fueron la excepción; en ellos se distinguen los valores otorgados a los habitantes de la frontera, que estaban en contraposición al discurso hegemónico de las élites de Medellín.

Finalmente, una forma de control social fue el higienismo, que moldeó los discursos de la antioqueñidad. La idea de una población civilizada fue apoyada por programas de higiene social que impulsaron los grandes empresarios con el fin de

22. Anexos al Informe del Ministro de Economía Nacional presentado al congreso de 1944, t. 3 (Bogotá: Imprenta Oficial, 1944) 168.

23. Eduardo Berrío González, Antioquia. Informe de la Secretaría de Gobierno (Medellín: Imprenta Departamental, 1946) 27.

24. Agosto 8 de 1932. Archivo Histórico de Antioquia (AHA), Medellín, Secretaría de Gobierno, Gobierno Municipios, t. 401, Segovia, f. 275r. 
generar un "comportamiento adecuado" en los residentes de Medellín y del departamento en general. ${ }^{25}$ Así, se reconocía la importancia de la higiene y la salud, "[que] es la base de la cultura y de la economía de un pueblo; del mismo modo que la vida es la síntesis y expresión del más alto valor digno de tal nombre... Si queremos alta moralidad y sólida cultura, luchemos porque los individuos crezcan sanos". ${ }^{26}$

Estas formas de intervención del centro en la periferia se basaron en primer lugar en lo económico y la principal expresión de esto fueron las obras de infraestructura y la consolidación de haciendas. La culminación de la línea férrea en 1929 y la construcción de la Troncal de Occidente buscaban anexar estas zonas al centro del departamento. Asimismo, el establecimiento de haciendas ganaderas permitió la diversificación de los negocios de élites comerciales de Medellín y su presencia en el Magdalena Medio y Nordeste antioqueños. A nivel político, la injerencia de la gobernación, por medio del nombramiento de alcaldes y las visitas administrativas, a la par del discurso higienista, se constituyeron en mecanismos importantes de centralización. No obstante, los discursos construidos por los grupos privilegiados tuvieron un fuerte componente racial que se basaba en la "antioqueñidad", la cual veía a los habitantes de zonas periféricas como incivilizados, atrasados, perezosos, de moral relajada y adscritos al partido liberal. Por tanto, estos individuos eran la antítesis del "antioqueño", que se consideraba más blanco, civilizado, trabajador y con valores morales conservadores, basados en la religión católica. Como se verá en los próximos acápites, la intervención del Estado regional se transformaría con la llegada de La Violencia. Hasta este momento, la intervención por medio de la fuerza ejercida por la policía departamental fue débil. El mismo visitador White Uribe advertía que la corrección de las conductas y la moralización de los habitantes de la periferia no debía hacerse por medio de la fuerza, sino por medio del "ejercicio de un verdadero y paciente apostolado". ${ }^{27}$ Así, la Iglesia debía adquirir un rol protagónico en la cohesión social de todo el departamento.

\section{El Bogotazo y las Juntas Revolucionarias en Segovia, Puerto Berrío y Remedios}

El gaitanismo, como se conoció al movimiento liderado por Jorge Eliécer Gaitán, significó la participación e influencia de sectores populares en la política colombiana. Este movimiento fue particularmente fuerte entre los obreros y artesanos urbanos (organizados y no organizados). Sin embargo, resalta su carácter multiclasista, ya que en él adquirieron protagonismo profesionales y comerciantes, algunos

25. Tubb 632 .

26. Antioquia. Informe de la Secretaría de Gobierno (Medellín: Imprenta Departamental, 1934) 202-204.

27. Guillermo White Uribe, "Concepto", 3 de diciembre de 1945. AHA, Medellín, Secretaría de Gobierno, Gobierno Municipios, t. 516, Segovia, carpeta 2, ff. 242r-245r. 
miembros de la política, pequeños propietarios rurales, trabajadores agrícolas y colonos. $^{28}$

Las primeras interpretaciones acerca del fenómeno de La Violencia subvaloraron el carácter del gaitanismo como una simple variante dentro del partido liberal. No obstante, este fue un proyecto antioligárquico, alternativo a los dos partidos tradicionales que tuvo además hondas repercusiones en La Violencia, ya que en algunas regiones esta se dirigió no solamente contra el partido liberal, sino contra de los bastiones gaitanistas. ${ }^{29}$

Este movimiento se asemejaría a los proyectos populistas que se dieron en la región entre 1930 y 1960, cuando se instaló el modelo de sustitución de importaciones y se dio un empuje mayor a la industrialización. Sin embargo, el gaitanismo posee varias particularidades que en ocasiones lo alejan de los populismos latinoamericanos. Cualquier caracterización del populismo en América Latina incluye un entorno urbano, una base social pluriclasista y una ideología con un tinte nacionalista. ${ }^{30}$ De ahí que el movimiento liderado por Gaitán sea comparable a otras experiencias populistas.

John Green concibe el gaitanismo como la expresión máxima del liberalismo de izquierda. Para el historiador norteamericano, este movimiento representó el surgimiento significativo de la participación popular en la política nacional. Gaitán asumió las aspiraciones y demandas de muchos colombianos que se encontraban en la periferia del poder para configurar un movimiento masivo de movilización radical que afectó las estructuras de poder. Así, el gaitanismo se apartaría del tipo de movilizaciones controladas que frecuentemente se asocian al populismo. Además, debido a la muerte temprana del líder, no gozó del poder político nacional. ${ }^{31}$

Los estudios sobre gaitanismo se debaten entre si se trató o no de una experiencia populista debido a la movilización de sectores no solamente urbanos, entre los que caben las clases medias, obreras y sectores campesinos. Para Ricardo López, son las clases medias las que hacen del gaitanismo un movimiento dificil de categorizar, ya que su participación hace de este un fenómeno diferente dentro de las experiencias populistas latinoamericanas. ${ }^{32}$

La discusión teórica es fundamental; pese a ello, aquí interesa resaltar el significado de la muerte de Gaitán, particularmente en las zonas de frontera del departamento de Antioquia. El Bogotazo está asociado a la insurrección que tuvo su epicentro en Bogotá, sin embargo, no se puede soslayar su impacto en otros lugares del país. Fuera de la capital, la muerte de Gaitán supuso la visibilización de

28. W. John Green, Gaitanismo, liberalismo de izquierda y movilización popular (Medellín / Bogotá: Fondo Editorial Universidad Eafit / Banco de la República, 2013) 33.

29. Sánchez, "Los estudios sobre la violencia” 27.

30. W. John Green, "Nuevas interpretaciones del populismo latinoamericano y el caso del gaitanismo en Colombia", Innovar. Revista de Ciencias Administrativas y Sociales 5 (1995): 119-125.

31. Green, "Nuevas interpretaciones" 124.

32. Ricardo López, “'Nosotros también somos parte del pueblo’: gaitanismo, empleados y la formación histórica de la clase media en Bogotá, 1936-1948”, Revista de Estudios Sociales 41 (2011): 90. 
la capacidad de diferentes sectores para la acción revolucionaria, lo que se tradujo no solamente en la conformación de "juntas revolucionarias", sino también en la incorporación de campesinos en las milicias populares que surgieron en aquel clima revolucionario. ${ }^{33}$

Las zonas de colonización de la primera mitad del siglo XX, correspondientes a las subregiones de Magdalena Medio, Bajo Cauca, Nordeste y Urabá, se constituyeron como regiones abrumadoramente liberales; aquí las ideas de Gaitán pudieron calar de manera más profunda que en otros municipios antioqueños, no solo por la predominancia del partido liberal, sino también porque el discurso gaitanista tenía fuertes connotaciones raciales y de clase. Para Green, las cuestiones de raza en relación con este movimiento son fundamentales, ya que las zonas predominantemente gaitanistas fueron justamente los lugares marginales poblados por mestizos, negros e indígenas. ${ }^{34}$ Gaitán capitalizó su experiencia como otro marginado racial, lo cual tuvo resonancia en el pueblo, que pensaba que, gracias a sus orígenes, este político nunca le daría la espalda. En consonancia con esta idea, zonas antioqueñas en donde Gaitán tuvo un mayor número de adeptos coinciden con los lugares que históricamente han sido vistos como racialmente "inferiores". En los municipios de frontera, los trabajadores petroleros y los portuarios estaban organizados. En Puerto Berrío, el Sindicato de Braceros se conformó en 1934, lo que permitió formalizar su trabajo. ${ }^{35}$ De igual manera, en Segovia, los mineros no solo de la Frontino Gold Mines, sino también de la Pato Gold Mines en Zaragoza, estaban organizados y respaldaron en 1946 la candidatura a la presidencia de Gaitán. En Zaragoza y Puerto Berrío, Gaitán recibió el mayor número de votos de Antioquia. ${ }^{36}$ Fue en estos municipios, y no en Medellín, en donde el gaitanismo tuvo mayor arraigo, especialmente tras el triunfo del conservador Mariano Ospina, cuando los despidos y hostigamientos por parte de funcionarios conservadores se hicieron más evidentes. ${ }^{37}$

Las fuentes primarias encontradas en referencia al 9 de abril de 1948 recopilan distintos relatos de lo ocurrido ese día y ponen de manifiesto la participación de varios sectores. Los más detallados hacen referencia a los sucesos de Puerto Berrío. Allí, de manera similar a Medellín, se armaron juntas revolucionarias y se atacaron negocios. Las impresiones de algunos visitantes de Medellín al puerto en aquella fecha quedaron registradas en el relato "Lo que vimos en Puerto Berrío"; según

33. Gonzalo Sánchez, Los días de la revolución. Gaitanismo y 9 de abril en provincia (Bogotá: Centro Cultural Jorge Eliécer Gaitán, 1983) 19.

34. Green, Gaitanismo, liberalismo de izquierda 215.

35. León Arredondo, "Liberalism, Working-class Formation and Historical Memory: Dockworkers in a Colombian Frontier" (Tesis de doctorado en Filosofia, The City University of New York, 2005) 134.

36. Mary Roldán, "Limitaciones locales de un movimiento nacional: Gaitán y el gaitanismo en Antioquia", Análisis Político 39 (2000): 28. Sobre los resultados de las elecciones presidenciales de 1946 véase Anuario Estadístico de Antioquia (1947).

37. Roldán, "Limitaciones locales" 28. 
ellos, después de que llegó la noticia de la muerte de Gaitán el viernes en la tarde, "el pueblo se sublevó y en el desorden fueron saqueados y destruidos por la turba cuatro establecimientos: la farmacia de unos señores Pinillos, y un bar de los mismos, la prendería de un señor Restrepo y el almacén de don Juancho Arango" 38

Los primeros ataques se centraron en los negocios de los conservadores, incluso Juan de Dios Arango - hijo de uno de los vecinos notables de Puerto Berrío, propietario de fincas ganaderas y negocios en el pueblo, y caracterizado por ser un conservador moderado - fue atacado por los gaitanistas. ${ }^{39}$ Ese mismo día, el pueblo, armado con machetes, tomó el casco urbano del puerto y se embriagó. Según los testigos, el Bogotazo en Puerto Berrío dejó 16 heridos y un muerto. Los días siguientes a la muerte de Gaitán transcurrieron en una tensa calma, y se afirma que los mismos habitantes del pueblo habían rechazado enérgicamente los ataques ocurridos en los comercios de los conservadores.

Asimismo, varios de los liberales del pueblo conformaron una Junta Revolucionaria que desconoció a las autoridades establecidas legalmente, y procedió al encarcelamiento del alcalde, Luis Cárcamo, quien fue trasladado a la cárcel de Puerto Olaya. ${ }^{40}$ Los miembros de la Junta intentaron mantener el orden público, y una de las medidas en este sentido fue la prohibición de la venta de licores durante el fin de semana. Sin embargo, esta supuesta calma pareció romperse con la llegada del ejército nacional, que arribó al pueblo al siguiente lunes. En ese momento empezaron a ser expedidos salvoconductos, sin los cuales los habitantes del pueblo no podían circular, pues serían encarcelados y tachados de "nueveabrileños".

Del mismo modo que en Puerto Berrío, en los municipios mineros de Segovia y Remedios también se conformaron Juntas Revolucionarias. En Segovia, inmediatamente se supo la noticia del asesinato de Gaitán, se conformó una junta que impidió que se entregara el despacho de la alcaldía al comandante de las fuerzas departamentales. Así, varios empleados pertenecientes a la rama judicial del municipio removieron al alcalde titular y desconocieron cualquier orden emanada por él. La Junta estaba conformada por varios funcionarios y vecinos pertenecientes al directorio municipal del partido liberal y al concejo municipal, y sus miembros pidieron la renuncia de otros funcionarios, entre ellos el administrador de hacienda y el vigilante de la policía municipal, ambos de filiación conservadora. Esto lo hicieron de la mano de los guardias departamentales, quienes eran liberales. De igual forma, la Junta propuso atacar a los conservadores del pueblo, sin embargo, esa propuesta no fue aceptada; en cambio, resolvieron cortar las líneas radiotelegráficas y destruir el campo de aterrizaje de Otú, ubicado cerca de Remedios. ${ }^{41}$

38. "Lo que vimos en Puerto Berrío", 1948. AHA, Medellín, Secretaría de Gobierno, Gobierno Municipios, t. 538, Puerto Berrío, f. 42.

39. "Lo que vimos en Puerto Berrío", f. 42.

40. Arredondo.

41. Fernando Uribe, "Informe que rinden los alcaldes: Segovia, Zaragoza y Remedios, de los acontecimientos subversivos de orden público", 1948. AHA, Medellín, Secretaría de Gobierno, Gobierno Municipios, t. 539, Segovia, carpeta 2, f. 57. 
Los revolucionarios segovianos se reunieron con los de Remedios en las dependencias de la Frontino Gold Mines. Este grupo estaba conformado por varios liberales remedianos, agentes de policía departamental y un alto número de particulares. Al ingresar a Remedios, subieron armados al despacho del alcalde para que hiciera entrega de la oficina y del cargo, mientras que en el salón del concejo municipal se realizaba una reunión, en la cual se constituía la nueva Junta Revolucionaria y se hacía la posesión solemne del nuevo alcalde. Particularmente en el caso de Remedios, el pueblo se congregó en la plaza principal para escuchar a los nuevos gobernantes locales, quienes anunciaron "el triunfo de la revolución” y el "derrumbamiento del gobierno conservador". Instalados en los balcones de la casa consistorial, llamaron a

La masacre, al incendio, al saqueo, e invitando al pueblo liberal a permanecer armado, ordenando la requisa rigurosa de los conservadores [...] exhortaba al pueblo a que amolara sus machetes, a que afilara sus puñales, para asesinar, para lapidar a los conservadores, ya que esa era la consigna ineludible de la revolución para vengar la muerte del doctor Gaitán. ${ }^{42}$

Cada uno de estos relatos presenta diferentes niveles de participación de los sectores involucrados en el Bogotazo, además de distintos grados de uso de la violencia. De acuerdo con las fuentes, la insurgencia en Remedios adquirió un impacto mayor, no solo por el número de funcionarios involucrados, sino también porque varios de los participantes del Bogotazo en Segovia se reunieron con los remedianos, con el fin de destruir el campo de aterrizaje de Otú e impedir la llegada de tropas del ejército por vía aérea. Aquí no solo hubo destrucción de los negocios de conservadores, sino que la Junta hizo un claro llamado a perseguir a todos los conservadores del pueblo.

En los relatos del Bogotazo en los municipios de Puerto Berrío, Segovia y Remedios, llaman la atención varios asuntos. En primer lugar, en los tres pueblos se establecieron gobiernos de facto, que fueron dirigidos por algunos funcionarios locales y miembros de los directorios municipales del partido liberal. En estas localidades hubo un claro acto de resistencia frente al gobierno legítimo, no solo departamental sino nacional este último liderado por Mariano Ospina, sobre quien se emprendió "una campaña de difamación”. La conformación de las Juntas Revolucionarias pone de manifiesto las pugnas entre las autoridades departamentales, que veían de manera prejuiciosa a quienes habitaban las zonas de frontera, por ser poblaciones étnicamente diferentes, y los políticos locales, que rechazaban a quienes ocupaban cargos impuestos por la gobernación. En este sentido, los alcaldes enviados desde Medellín reforzaban las visiones de las élites, ya que calificaban a los gaitanistas como un grupo de "agitadores políticos" y "revoltosos", que respaldaban "descabelladas e infames actividades contra el orden y la tranquilidad 
social". ${ }^{43}$ Otro aspecto importante tiene que ver con la participación de agentes de policía. Tanto en Segovia como en Puerto Berrío, los agentes mostraban una actitud favorable hacia las actuaciones de "nueveabrileños", e incluso eran partícipes de las acciones tomadas por las Juntas. Así, tanto funcionarios civiles como agentes de policía se unieron para excluir a cualquier miembro del partido conservador de la administración local, con lo que reforzaron su identidad liberal y gaitanista.

Cuando pasó la efervescencia del Bogotazo, y en los meses posteriores, desde el gobierno departamental se tomaron medidas con el fin de reprimir a quienes habían conformado las Juntas Revolucionarias. Esto se expresó por medio de la llegada de alcaldes conservadores y militares que pudieran resguardar el orden público. En Remedios, de la misma manera que como se había hecho en años anteriores, los vecinos solicitaban el cambio de alcaldes a través de memoriales. No obstante, la diferencia respecto de las anteriores peticiones radicaba en que los firmantes formaron parte de las Juntas Revolucionarias; es decir, los liberales del pueblo eran quienes se quejaban del sectarismo y de la persecución hacia ellos:

Cuando no es que somos conducidos a la cárcel, brutalmente, a los bayonetazos e insultos proferidos por los agentes de policía [...] se nos requisa llegando hasta el punto de violar nuestros domicilios, amenazando a nuestras familias [...] ¿Todo por qué? ¿Por la única causa de ser liberales $[\ldots]$ ¿por qué se nos persigue tan vilmente? ${ }^{44}$

Esta primera manifestación de violencia en las zonas periféricas presenta la visión del Bogotazo desde la región. Las fuentes documentales en torno a este suceso permiten reconstruir el relato de lo ocurrido en estos municipios alejados del poder central; con base en ellas, es posible observar que los hechos de violencia no se presentaron con la misma intensidad. En estas subregiones, la insurgencia se localizó en los municipios de Puerto Berrío, Remedios y Segovia; el primero de ellos tenía una importante tradición liberal y, desde la década de los cuarenta, gaitanista, y los dos últimos eran importantes distritos mineros liberales que resistieron al poder departamental por medio de Juntas Revolucionarias, lo que se tradujo en el reforzamiento de las visiones desde el centro, que apuntaban a ver a los locales como insubordinados y rebeldes que desacataban el orden impuesto desde el partido conservador.

\section{La Violencia en Antioquia: del Bogotazo al Frente Nacional}

Después del Bogotazo, los conflictos siguieron manifestándose a través de las disputas entre lugareños y funcionarios designados por el gobierno departamental. No obstante, desde 1949 empezaron a registrarse algunos hechos de violencia que

43. Alcaldía Municipal, 11 de abril de 1948, f. $121 \mathrm{r}$.

44. 11 de julio de 1948. AHA, Medellín, Secretaría de Gobierno, Gobierno Municipios, t. 538, Remedios, f. 153 r. 
se expresaron por medio de asaltos en haciendas cuyos propietarios eran conservadores. Estos ataques aumentaron y en 1951 se sumó a los asaltos el asesinato de campesinos. Los ataques fueron perpetrados por guerrilleros que venían del otro lado del río Magdalena. Las primeras guerrillas identificadas fueron conformadas en los departamentos de Santander y Boyacá y se desplazaron hacia el oeste, hasta llegar al departamento de Antioquia. ${ }^{45}$

A nivel nacional la fase de La Violencia que va de 1949 a 1953 estuvo marcada por el ascenso al poder de Laureano Gómez, una figura relativamente nueva dentro del conservatismo y en torno a la cual se consolidó una facción extremista del partido conservador. Durante esos años, La Violencia se extendió al mundo rural, transformándose en una confrontación armada entre guerrillas liberales y grupos de conservadores, popularmente conocidos como "chusmeros" y "contrachusmeros", respectivamente. ${ }^{46}$ De acuerdo con Mary Roldán, en Antioquia esta fase se expresaría de manera violenta a partir de 1952, cuando ya operaban guerrillas propiamente antioqueñas. ${ }^{47}$ Sin embargo, desde 1951 había ataques de guerrilleros liberales, auspiciados por algunos gamonales, comerciantes y políticos de Puerto Berrío y Remedios. Roldán analiza esta región del oriente de manera general, pero, al evaluar de cerca la documentación, se pone de manifiesto que aquí también hubo una geografia de la violencia, donde unos municipios tuvieron un mayor número de víctimas y donde hubo predominancia de campesinos conservadores o liberales en armas dependiendo del municipio.Ver de cerca la dinámica del conflicto permite identificar a ciertos personajes locales, adscritos en su mayoría al partido liberal, quienes no solamente solventaron las bandas, sino que también fueron protagonistas en la formación de redes de poder local adscritas al liberalismo, las cuales se vieron amenazadas con la llegada de los conservadores.

El escenario principal de LaViolencia fueron las haciendas de empresarios agrícolas y ganaderos; por esa razón, algunos de ellos rechazaron los hechos violentos y presionaron a los gobernadores durante la década de los cincuenta para que se pusieran en marcha planes de pacificación para recuperar la productividad disminuida a causa de la violencia. A partir de 1953, las comisiones de orden público irían en aumento, además de la conformación de los comités propaz encabezados por varios vecinos notables de "ambas colectividades", que buscaban frenar la ola de asesinatos y otros actos violentos.

Los asaltos se dieron principalmente en las fincas de conservadores; en ellas asesinaban o secuestraban a quienes no eran "adictos a la chusma" o al partido liberal. En este primer momento, los ataques guerrilleros eran puntuales y mayormente identificables por parte de agentes de la policía y el ejército. Así, la llegada de La Violencia hizo que la intervención estatal se hiciera por medio de la fuerza, a través

45. Roldán.

46. Diana Henao Holguín, “"Ni Robín de los bosques, ni vengador a sangre'. Bandolerismo rural en Antioquia, 1953-1965”, Escrita con sangre. Historia de la violencia en América Latina: siglos XIX Y XX, comps. Igor Goicovic y otros (Santiago de Chile: Ceibo Ediciones, 2013) 149-179.

47. Roldán, $A$ sangre y fuego 158. 
de policías departamentales y, a partir de 1953, por medio de comisiones de orden público a cargo de militares.

Un caso que puede ilustrar las agresiones de guerrilleros liberales a fincas de empresarios de Medellín es el ataque a la hacienda ganadera Ité, propiedad de la familia Bedout, ${ }^{48}$ el 3 de enero de 1951, lo que dejó como resultado el asesinato de diez campesinos, cuatro de ellos menores de edad. En este caso, comisiones de orden público fueron enviadas el mismo día desde Antioquia y Santander; siguieron el rastro de los guerrilleros liberales, identificaron a varios de los involucrados en el asesinato de los campesinos e incluso capturaron a dos de ellos. En este primer momento, hubo una mayor coordinación entre las brigadas del ejército y un seguimiento más cercano de las bandas. Además, parece que la presencia de militares fue más fuerte en las haciendas, con el fin de "prestar protección a los ciudadanos y batir a los bandoleros". ${ }^{49}$

Estos ataques se caracterizaron por el alto grado de violencia, expresada a través de una estrategia de erradicación humana intensiva. Esta violencia se concentró en las haciendas, aunque también ocurrió en algunas minas y, a partir de 1953, se registraron varios ataques en las compañías extranjeras, principalmente en la Frontino Gold Mines, donde había un mayor número de pertrechos para la operación de las bandas, como armas, dinamita y alimentos.

Los ataques en un primer momento se dieron especialmente en las fincas de los conservadores. No obstante, estas no sufrieron solamente la violencia de los guerrilleros liberales, sino también de los ejércitos privados de conservadores (“contrachusmeros"). En este sentido, el asalto a una de las fincas de Juan de Dios Arango es ilustrativo. Este ganadero y comerciante, además de ser un vecino notable de Puerto Berrío, era un importante miembro del partido conservador. Los ataques ocurrieron en su finca, ubicada en el corregimiento de Virginias, al lado de la estación Palestina del Ferrocarril, en la zona correspondiente a los pasajes El Vapor y Cominal.

La particularidad de este asalto es que estuvo en manos de un ejército privado de conservadores que entró a buscar liberales en una hacienda de Puerto Berrío. De acuerdo con el informe realizado por el inspector de policía de Virginias, en la mañana del 18 de mayo de 1952 se presentó en la finca San Francisco "una cuadrilla de malhechores en número no menor de treinta" armada con escopetas, peinillas y cuchillos. La noche anterior habían pasado por la finca La Soledad, propiedad de Octavio y Francisco Ríos; aquí requisaron la casa del mayordomo en busca de medicinas, dinero y comida y "una vez ellos comieron se fueron en dirección hacia la mina el Concejo", donde asesinaron a varios mineros que estaban

48. Bedout fue una reconocida familia que ayudó a consolidar la industria de la litografía y la imprenta en Colombia. El éxito de este negocio le permitió diversificar sus actividades e invertir en la ganadería, por eso desde la década de los treinta varios de sus miembros empezaron a hacer denuncios en Puerto Berrío y Remedios para conformar haciendas ganaderas.

49. Jaime Hoyos Trujillo, "Situación de orden público", 13 de enero de 1951.AHA, Medellín, Secretaría de Gobierno, Gobierno Municipios, t. 564, Puerto Berrío, f. 8. 
en el campamento. Cuando llegaron a El Vapor, reunieron a varios de los dueños de las casas de habitación del paraje, quienes fueron amarrados y posteriormente asesinados.

En relación con el conflicto político, es importante mencionar que ya para 1952, los ataques a las haciendas no eran exclusivos de los guerrilleros liberales; en este caso, si bien los campesinos identificaron la banda con los "chusmeros" o "bandidos", los hombres armados especificaron que iban en búsqueda de "cachiporros", "manzanillos" o liberales, y a los únicos campesinos que no asesinaron fue a los que se identificaron oficialmente como militantes del partido conservador. De esta forma, los ejércitos privados de conservadores empezaron a tener una mayor participación en esta fase violenta y se enfrentaron de manera más recurrente a los ejércitos liberales.

Los intereses de los empresarios agrícolas de estas zonas se vieron seriamente afectados por la ola de violencia que vivía la región, no solo porque los guerrilleros y "contrachusmeros" asaltaban las haciendas, sino porque los campesinos estaban abandonando los campos, por lo que la consecución de mano de obra se hacía cada vez más compleja. En este contexto, los hacendados y las autoridades buscaron implementar varias medidas que frenaran los ataques dentro de las fincas, entre las que destacan el chequeo de los trabajadores y la expedición de salvoconductos a quienes no tuvieran asuntos pendientes con la justicia. Estas medidas buscaban ejercer control sobre quienes trabajaban en las haciendas para evitar que "chusmeros" o "bandidos" se infiltraran en ellas. Según los dueños de las haciendas y las autoridades locales, se debía tener trabajadores leales, puesto que eran estos quienes indicaban a las chusmas "aquellos elementos adictos al gobierno", además de que abastecían a los guerrilleros de armas y provisiones y muchas veces ingresaban a las filas de las bandas.

Esto no solamente se daba en haciendas de conservadores, sino de liberales que no estaban de acuerdo con la "chusma". Por ello, en muchas ocasiones se optó no solo por solicitar la presencia de agentes del gobierno en las haciendas, sino que se pedían armas para que los trabajadores se defendieran de los ataques. Los empresarios pedían al gobernador: "si no tiene hombres suficientes, al menos nos de armas para nosotros mismos ayudarle a defender la región". ${ }^{0}$ No obstante, la conformación de ejércitos privados en las haciendas no fue exclusiva de esta época; desde antes de 1948, varios empresarios agrícolas optaron por el uso de este tipo de seguridad privada.

Esta primera ola de violencia en el Magdalena Medio y el Nordeste antioqueños se caracterizó por ser más tardía que en otras regiones del país, ya que se intensificó a finales de 1951, cuando los ataques de guerrilleros liberales se dieron en fincas y el asesinato de campesinos se hizo sistemático, con lo que inició su desplazamiento hacia los cascos urbanos. Como se observa, los guerrilleros liberales fueron un

50. "Declaración", 1953. AHA, Medellín, Secretaría de Gobierno, Gobierno Municipios, t. 589, Puerto Berrío, f. 109v. 
actor fundamental; si bien se reconoce que la conformación de ejércitos privados de conservadores o "contrachusmeros" incrementó la violencia, paralelamente las guerrillas liberales, venidas desde Boyacá y después propiamente antioqueñas, la incentivaron.

Por otra parte, las medidas propuestas para la "pacificación" se vieron entorpecidas por las autoridades de los municipios, cuya incompetencia era denunciada por los alcaldes militares. Sobre los agentes de policía en Segovia se decía que:

Son en su mayoría borrachos empedernidos, ignorantes o lo que es lo mismo sin ninguna preparación intelectual y lo que es peor sin ningún conocimiento sobre procedimientos, son muchachos de diez y nueve a veintidós años de edad a quienes no les importa más que enamorar, tomar trago en uniforme, son tímidos, y se dejan comprar hasta por una botella de cerveza. ${ }^{51}$

En este sentido, los militares asociaban la juventud segoviana con la embriaguez y la falta de preparación intelectual; además, cabe recordar que varios policías locales participaron en el Bogotazo. En contraposición, se encontraba la visión de los agentes de policía municipales, quienes se quejaban de las autoridades militares y sus procedimientos, con lo que se perciben las tensiones entre autoridades civiles y militares locales y departamentales:

Es verdaderamente lamentable y un desprestigio para nuestro Gobierno, los procederes de la comisión de orden formada por unidades de la Policía Militar... entra a los campos infundiendo el terror a labriegos honrados; hurtan todo lo que encuentran a su paso; ponen prisioneros a hombres honrados y en general cometen toda clase de abusos y arbitrariedades. ${ }^{52}$

Paralelamente al uso de la fuerza, a nivel institucional se decretó la amnistía durante el gobierno de Gustavo Rojas Pinilla, con el fin de reincorporar a los guerrilleros a la vida civil. Asimismo, se crearon centros de rehabilitación para prestar ayuda a los desplazados de la violencia y a los guerrilleros que voluntariamente entregaran sus armas ante las autoridades militares o la policía nacional. En estos centros se debía dar ayuda material y brindar garantías de seguridad, tanto a los guerrilleros como a los campesinos damnificados. ${ }^{53}$

A nivel nacional, estas medidas se tradujeron en la creación de algunas instituciones que pretendían solucionar el problema de la tierra, una de las causas fundamentales del conflicto. De esta manera, se crearon el Instituto de Parcelaciones, Colonización y Defensa Forestal (1948), el Instituto de Colonización e Inmigración (1953) y la Oficina de Rehabilitación y Socorro (1953). También, durante el Frente Nacional, Alberto Lleras Camargo impulsó el Plan Nacional de

51. "Informe privado o confidencial", 29 de mayo de 1955. AHA, Medellín, Gobierno Ramos, Despacho del Gobernador, DG 84, f. 242.

52. AHA, Medellín, Secretaría de Gobierno, Gobierno Municipios, t. 564, Segovia, f. 137r.

53. "La vuelta al orden y la tranquilidad abren perspectivas a la economía”, El Correo (Medellín) 8 de agosto de 1953: 1. 
Rehabilitación (1958), que, de manera similar a la Oficina de Rehabilitación y Socorro, buscaba intervenir las zonas afectadas por la violencia. ${ }^{54}$ Es así como desde 1948, y hasta la conformación del Frente Nacional (1958), los distintos gobiernos se encaminaron en la búsqueda de la "pacificación", no solo a través de mecanismos violentos, sino mediante la institucionalidad. Estas instituciones, si bien buscaban "rehabilitar" las zonas afectadas por la violencia, auxiliando a los exiliados y damnificados en las regiones azotadas por la violencia, también tenían por objetivo levantar las estadísticas de tierras abandonadas y de los bienes y negocios destruidos. ${ }^{55}$ En consecuencia, buscaban mitigar el impacto del desplazamiento y promover el retorno de campesinos a sus tierras.

Con estas medidas, el gobierno departamental y nacional suponía que la calma retornaría a los campos. Sin embargo, el periodo que va de 1953 a 1958 estuvo caracterizado por la proliferación de grupos de bandoleros, ya que los guerrilleros amnistiados en vez de retornar al trabajo en los campos, rápidamente se rearmaron y siguieron operando. Por ello, la presencia militar en las zonas de frontera continuó. De manera similar a los visitadores administrativos de los gobiernos civiles anteriores, los militares reprodujeron miradas prejuiciosas de los habitantes en la periferia:

La región de Puerto Berrío o más claro, las laderas del río Magdalena está poblada la mayor parte por elementos indeseables porque allí se han refugiado maleantes, prófugos de la justicia, ladrones, asesinos, etc. Estos sujetos de pésima conducta se convierten allí en colonos, trabajadores de las fincas, mayordomos y braceros, actividades con las cuales disfrazan muchas veces sus incursiones fuera de la ley. Entre el personal de las fincas el más peligroso es el de trabajadores flotantes, ya que su movilidad, su inestabilidad y demás circunstancias del trabajo que desempeñan los hace esencialmente aptos para servir como miembros, agentes, informantes, espías y comisionados de los bandoleros... Los braceros son personal de lo peor que existe en la región. Como viven permanentemente en los poblados, son especiales para servir de enlace entre los bandoleros y los patrocinadores que permanecen ocultos. Como son por regla general comunistas o filocomunistas, tienen especial empeño en fomentar actos subversivos y situaciones de violencia. ${ }^{56}$

Así, los militares enviados a las zonas de frontera veían imposible el retorno a la paz, dado el carácter de quienes allí habitaban, ya que eran simpatizantes de los bandoleros. Por ello, la intervención estatal entre 1953 y 1958 se basó en buena medida en el uso de la fuerza. No obstante, también hubo otros mecanismos institucionales para la intervención en la frontera y la resolución del conflicto, que se

54. Sobre la Comisión Investigadora de 1958 y el significado del estudio sobre la Violencia en Colombia véase Jefferson Jaramillo Marín, "La Comisión Investigadora de 1958 y la Violencia en Colombia”, Universitas Humanística 72 (2011): 37-62; Robert A. Karl, Forgotten Peace: Reform, Violence, and the Making of Contemporary Colombia (Oakland: University of California Press, 2017).

55. "Decreto 2466 de 1953 (septiembre 22) por el cual se señalan las funciones de la Oficina de Rehabilitación y Socorro y se dictan otras disposiciones”, Diario Oficial (Bogotá) 2 de octubre de 1953: 2.

56. AHA, Medellín, Gobierno Ramos, Despacho del Gobernador, DG 41, ff. 272-278. 
expresaron principalmente en la aparición de instituciones que buscaban solucionar las consecuencias de La Violencia por medio de la "rehabilitación" económica del campo. De esta manera, el Estado se valió de la represión y los canales institucionales para frenar LaViolencia e intervenir en las zonas de frontera, en donde se vivió con más fuerza el fenómeno.

\section{Conclusiones}

Desde fines del siglo XIX, la política pública en Colombia intentó penetrar y hacer funcionar las zonas de frontera. Expresión de ello fueron la construcción de obras de infraestructura, como las líneas ferroviarias y carreteras y el despliegue de una profusa institucionalidad municipal (alcaldes y visitadores administrativos). Junto con ello es posible observar una extensión de las haciendas y un incremento de la colonización de baldíos. Estos fenómenos dieron origen a una serie de tensiones, entre las cuales destacan aquellas que confrontaron a caciques locales y autoridades estatales, hacendados y trabajadores agrícolas, Iglesia y feligresía y comerciantes y burocracia pública. En este contexto, el ideal modernizador del Estado aparecía fuertemente cuestionado desde la comunidad local. Esto favoreció la emergencia de un discurso oficial (higienista), patrocinado por el Estado, la Iglesia y las élites conservadoras, que afirmó que los problemas de la región de Antioquia (económicos, políticos, sociales y culturales) derivaban de la composición racial de la población (mestizos), factor que los inhabilitaba para ser trabajadores disciplinados y responsables y les impedía desenvolverse socialmente de acuerdo con las normas civiles y religiosas existentes.

El asesinato del líder liberal Jorge Eliécer Gaitán (1948) y la extensión del levantamiento popular hacia los departamentos y municipios de Colombia redoblaron el cuestionamiento a las autoridades del Estado. Los caciques locales, aliados con franjas significativas de las clases medias, de los trabajadores urbanos y del campesinado, formaron Juntas Revolucionarias en algunos casos o dieron origen a poderosas bandas guerrilleras que disputaron con el Estado el control de los territorios y, junto con ello, de las poblaciones. Ello exacerbó los enfrentamientos y las matanzas de civiles desarmados, con lo que escaló exponencialmente la violencia. A su vez, este cuestionamiento al monopolio de la violencia que debiera ejercer el Estado, aparejado al discurso aniquilador del adversario, fracturó el tejido social y favoreció la articulación de redes de poder fragmentarias.

Si bien el régimen de Gustavo Rojas Pinilla (1953-1957) logró controlar los fenómenos violentos a través de las comisiones militares desplegadas en los territorios más conflictivos, al dictar la Ley de Amnistía (1953) y con la creación de una institucionalidad preocupada de abordar el problema de acceso del campesinado a la tierra, la violencia se arraigó como práctica política y social y, en cuanto tal, favoreció la consolidación de los poderes caciquiles locales en detrimento del Estado. De esta manera, hacendados y gamonales no solo ostentaron el control de la violencia, sino que además nominaron a las autoridades políticas locales, direccionaron 
la inversión de las rentas públicas, planificaron la asignación de tierras, controlaron los destacamentos policiales y la administración de justicia y formaron redes de clientelas electorales.

\section{Fuentes}

\section{Manuscritas}

Archivo Histórico de Antioquia, Medellín (AHA)

Secretaría de Gobierno

Gobierno Ramos

\section{Impresas}

Anexos al Informe del Ministro de Economía Nacional presentado al congreso de 1944. Tomo 3. Bogotá: Imprenta Oficial, 1944.

Antioquia. Informe de la Secretaría de Gobierno. Medellín: Imprenta Departamental, 1934. Berrío González, Eduardo. Antioquia. Informe de la Secretaría de Gobierno. Medellín: Imprenta Departamental, 1946.

\section{Periódicos}

Diario Oficial (Bogotá) 1953.

El Correo (Medellín) 1953.

\section{Bibliografía}

Alba Vega, Carlos y Dirk Kruijt. "Viejos y nuevos actores violentos en América Latina: temas y problemas”. Foro Internacional 47.3 (2007): 485-516.

Álvarez Múnera, José Roberto. Mercado, ganado y territorio: haciendas y hacendados en el Oriente y el Magdalena Medio antioqueños (1920-1960). Medellín: Universidad de Antioquia / Editorial Universidad Pontificia Bolivariana, 2016.

Ansaldi, Waldo y Verónica Giordano. Coords. América Latina. Tiempos de violencias. Buenos Aires: Ariel, 2014.

Appelbaum, Nancy P. Muddied Waters: Race, Region, and Local History in Colombia, 1846-1948. Durham / Londres: Duke University Press, 2003.

Arredondo, León. "Liberalism, Working-class Formation and Historical Memory: Dockworkers in a Colombian Frontier". Tesis de doctorado en Filosofia, The City University of New York, 2005.

Betancourt, Darío y Martha García. Matones y cuadrilleros. Origen y evolución de la violencia en el occidente colombiano, 1946-1965. Bogotá: Tercer Mundo Editores, 1990.

Bolívar, Ingrid. Violencia política y formación del Estado. Ensayo historiográfico sobre la 
dinámica regional de la violencia de los Cincuenta en Colombia. Bogotá: Universidad de los Andes / CINEP, 2003.

Bravo Vargas, Viviana. "La sangre, la furia y la memoria: Ramona Parra en el martirologio comunista de la postguerra (Chile 1946-1947)”. Páginas 9.20 (2017): 32-52.

Cattaruzza, Alejandro. Historia de la Argentina, 1916-1955. Buenos Aires: Siglo XXI Editores, 2016.

Dunkerley, James. Rebelión en las venas. La lucha política en Bolivia, 1952-1982. La Paz: Plural Editores, 2003.

García Ferreira, Roberto. "La revolución guatemalteca y el legado del presidente Arbenz". Anuario de Estudios Centroamericanos 38 (2012): 41-78.

Goicovic, Igor y Jaqueline Vassallo. Comps. América Latina: violencias en la historia. Valparaíso: América en Movimiento, 2018.

González, Fernán. Poder y violencia en Colombia. Bogotá: Odecofi-CINEP, 2014.

Green, W. John. Gaitanismo, liberalismo de izquierda y movilización popular. Medellín / Bogotá: Fondo Editorial Universidad Eafit / Banco de la República, 2013.

. "Nuevas interpretaciones del populismo latinoamericano y el caso del gaitanismo en Colombia”. Innovar. Revista de Ciencias Administrativas y Sociales 5 (1995): 119-125.

Guzmán Campos, Germán, Orlando Fals Borda y Eduardo Umaña Luna. La violencia en Colombia: estudio de un proceso social. Bogotá: Ediciones Tercer Mundo, 1962.

Henao Holguín, Diana. " Ni Robín de los bosques, ni vengador a sangre'. Bandolerismo rural en Antioquia, 1953-1965”. Escrita con sangre. Historia de la violencia en América Latina: siglos XIX y XX. Comps. Igor Goicovic, Julio Pinto, Ivette Lozoya y Claudio Pérez. Santiago de Chile: Ceibo Ediciones, 2013.

Imbusch, Peter, Michel Misse y Fernando Carrión. "Violence Research in Latin America and the Caribbean: A Literature Review". International Journal of Conflict and Violence 5.1 (2011): 87-154.

Jaramillo Marín, Jefferson. “La Comisión Investigadora de 1958 y la Violencia en Colombia”. Universitas Humanística 72 (2011): 37-62.

Karl, Robert A. Forgotten Peace: Reform, Violence, and the Making of Contemporary Colombia. Oakland: University of California Press, 2017.

Kruijt, Dirk, Eduardo Rey Tristán y Alberto Martín Álvarez. Latin American Guerrilla Movements. Origins, Evolution, Outcomes. Nueva York: Routledge, 2020.

López, Ricardo. “'Nosotros también somos parte del pueblo’: gaitanismo, empleados y la formación histórica de la clase media en Bogotá, 19361948". Revista de Estudios Sociales 41 (2011): 84-105.

Oquist, Paul. Violencia, conflicto y política en Colombia. Bogotá: Biblioteca Banco Popular, 1978. 
Ortiz Sarmiento, Carlos Miguel. Estado y subversión en Colombia. La Violencia en el Quindío años 50. Bogotá: Cerec, 1985.

Pécaut, Daniel. Orden y violencia: Colombia 1930-1953. Medellín: Fondo Editorial Universidad Eafit, 2012.

Pozzi, Pablo y Claudio Pérez. Historia oral e historia política. Izquierda y lucha armada en América Latina, 1960-1990. Santiago de Chile: LOM Ediciones, 2012.

Rojas, Rafael. Historia mínima de la Revolución Cubana. Ciudad de México: El Colegio de México, 2015.

Roldán, Mary. A sangre y fuego. La Violencia en Antioquia, Colombia, 1946-1953. Bogotá: Instituto Colombiano de Antropología e Historia / Fundación para la Promoción de la Ciencia y la Tecnología, 2003.

."Limitaciones locales de un movimiento nacional: Gaitán y el gaitanismo en Antioquia”. Análisis Político 39 (2000): 17-35.

. "Violencia, colonización y la geografia de la diferencia cultural en Colombia”. Análisis Político 35 (1998): 3-25.

Sánchez, Gonzalo y Donny Meertens. Bandoleros, gamonales y campesinos. El caso de la Violencia en Colombia. Bogotá: Punto de Lectura, 2006.

Sánchez, Gonzalo. Los días de la revolución. Gaitanismo y 9 de abril en provincia. Bogotá: Centro Cultural Jorge Eliécer Gaitán, 1983.

. "Los estudios sobre la violencia: balance y perspectivas". Pasado y presente de la violencia en Colombia. Comps. Gonzalo Sánchez y Ricardo Peñaranda. Bogotá: Fondo Editorial Cerec, 1991.

Serje, Margarita. El revés de la nación: territorios salvajes, fronteras y tierras de nadie. Bogotá: Universidad de los Andes, 2011.

Steiner, Claudia. Imaginación y poder: el encuentro del interior con la costa en Urabá, 1900-1960. Medellín: Editorial Universidad de Antioquia / Ediciones Uniandes, 2019.

Tubb, Daniel. "Narratives of citizenship in Medellín, Colombia". Citizenship Studies 17.5 (2013): 627-640. DOI: 10.1080/13621025.2013.818380.

Wade, Peter. Gente negra, nación mestiza: dinámicas de las identidades raciales en Colombia. Bogotá: Siglo del Hombre Editores / Ediciones Uniandes, 1997. 